\title{
Time to abandon ampicillin plus gentamicin in favour of ampicillin plus ceftriaxone in Enterococcus faecalis infective endocarditis? A meta-analysis of comparative trials
}

\author{
Moritz Mirna $^{1}$ D $\cdot$ Albert Topf $^{1} \cdot$ Lukas Schmutzler $^{1} \cdot$ Uta C. Hoppe ${ }^{1} \cdot$ Michael Lichtenauer $^{1}$
}

Received: 1 August 2021 / Accepted: 3 November 2021 / Published online: 9 November 2021

(c) The Author(s) 2021

\begin{abstract}
Background Current guidelines recommend either ampicillin plus ceftriaxone (AC) or amoxicillin/ampicillin plus gentamicin (AG) with an equivalent class IB recommendation in Enterococcus faecalis endocarditis. However, previous observational studies suggest that $\mathrm{AC}$ might be favourable in terms of adverse events.

Objectives To investigate whether AC is non-inferior to AG, and if it is associated with less adverse events.

Methods In June 2021, a systematic literature search using the databases PubMed/MEDLINE, CDSR, CENTRAL, CCAs, EBM Reviews, Web of Science and LILACS was conducted by two independent reviewers. Studies were considered eligible if (P) patients included were $\geq 18$ years of age and had IE with E. faecalis, (I) treatment with AC was compared to (C) treatment with $\mathrm{AG}$ and $(\mathrm{O})$ outcomes on in-hospital mortality, nephrotoxicity and adverse events requiring drug withdrawal were reported. Odds ratios and 95\% confidence intervals were calculated using random-effects models with the Mantel-Haenszel method, the Sidik-Jonkman estimator for $\tau^{2}$ and the Hartung-Knapp adjustment.

Results Treatment with AC was non-inferior to AG, as depicted by no significant differences in-hospital mortality, 3-month mortality, relapses or treatment failure. Furthermore, AC was associated with a lower prevalence of nephrotoxicity (OR 0.45 [0.26-0.77], $p=0.0182$ ) and drug withdrawal due to adverse events (OR $0.11[0.03-0.46], p=0.0160$ ) than AG.

Conclusions Treatment with AC was non-inferior to treatment with AG, and it was associated with a reduced prevalence of nephrotoxicity and drug withdrawal due to adverse events. Thus, combination therapy with AC appears favourable over AG in patients with E. faecalis IE.
\end{abstract}

Moritz Mirna and Albert Topf have contributed equally to this work.

Moritz Mirna

m.mirna@salk.at

1 Division of Cardiology, Department of Internal Medicine

II, Paracelsus Medical University of Salzburg, Müllner

Hauptstraße 48, 5020 Salzburg, Austria 


\section{Graphical abstract}

\begin{tabular}{|c|c|c|}
\hline \multicolumn{3}{|c|}{$\begin{array}{l}\text { Time to abandon ampicillin plus gentamicin in favour of ampicillin plus ceftriaxone in } \\
\text { Enterococcus faecalis infective endocarditis? - a meta-analysis of comparative trials }\end{array}$} \\
\hline Methods & Results & Conclusion \\
\hline $\begin{array}{l}\text { - systematic literature research by } 2 \\
\text { independent reviewers } \\
\text { - OR and } 95 \% \mathrm{Cl} \text { calculated with random } \\
\text { effects models } \\
\text { P: } \geq 18 \text { years with E.faecalis IE } \\
\text { I: ampicillin + ceftriaxone (AC) } \\
\text { C: amoxi-/ampicillin + gentamicin (AG) } \\
\text { O: in-hospital mortality, nephrotoxicity, AE } \\
\text { requiring drug withdrawal }\end{array}$ & $\begin{array}{l}4 \text { retrospective, nonrandomized, observational } \\
\text { cohort studies included; total } N=599 \\
\text { - no significant differences in in-hospital } \\
\text { mortality, } 3 \text {-month mortality, relapses or } \\
\text { treatment failure between } A C \text { and } A G \\
\text { - AC with lower prevalence of nephrotoxicity } \\
\text { (OR: } 0.45 \text { [0.26-0.77], } p=0.0182 \text { ) } \\
\text { - AC with lower prevalence of drug } \\
\text { withdrawal due to adverse events (OR } 0.11 \\
\text { [0.03-0.46], } p=0.0160 \text { ) }\end{array}$ & $\begin{array}{l}\text { Treatment with } A C \text { was non-inferior to } \\
\text { treatment with } A G \text {, and it was } \\
\text { associated with a reduced prevalence } \\
\text { of nephrotoxicity and drug withdrawal } \\
\text { due to adverse events. } \\
\text { Thus, combination therapy with AC } \\
\text { appears favourable over AG in patients } \\
\text { with E.faecalis IE. }\end{array}$ \\
\hline
\end{tabular}

Keywords Cardiology $\cdot$ Endocarditis $\cdot$ Meta-analysis $\cdot$ Enterococcus $\cdot$ Ampicillin $\cdot$ Ceftriaxone $\cdot$ Gentamicin

\section{Introduction}

Due to its elaborated and tedious treatment regimens, as well as its associated morbidity and mortality, infective endocarditis (IE) frequently poses a challenge to the attending physician in clinical practice. Endocarditis can be caused by various different microorganisms, with Enterococci spp. representing the third most common group of pathogens, with an estimated frequency of $10 \%$ of all cases [1]. Among all cases of enterococcal IE, Enterococcus faecalis further represents the predominant strain of Enterococci, with a prevalence of almost $90 \%$ [2].

To date, the European Society of Cardiology (ESC) recommends both a combination of two cell wall inhibitors, namely ampicillin plus ceftriaxone for 6 weeks (AC), or one cell wall inhibitor with an aminoglycoside antibiotic, i.e., amoxicillin/ampicillin plus gentamicin for 2-6 weeks (AG), with equivalent class IB recommendations for the treatment of E. faecalis IE [3]. However, alarmingly high rates of highlevel aminoglycoside-resistant strains of $E$. faecalis and $E$. faecium (HLAR; aminoglycoside Minimal Inhibitory Concentration $(\mathrm{MIC})>500 \mathrm{mg} / \mathrm{L}$ ) have been reported in previous studies $[2,4,5]$, which may preclude the proclaimed synergistic bactericidal effects of a combination of cell wall inhibitor plus aminoglycoside [6].

Furthermore, treatment with aminoglycosides is associated with an increased risk for adverse effects, such as drug-induced nephrotoxicity or ototoxicity [7-9], and the dosing and monitoring of these antibiotics can be quite challenging in clinical practice, especially in patients with already impaired kidney function, elderly or obese patients $[10,11]$.

Previous observational studies reported that combination therapy with $\mathrm{AC}$ could be non-inferior to treatment with AG in patients with E. faecalis IE in terms of mortality and relapses, as well as beneficial regarding the risk for drug-induced adverse events [12-14]. However, there is currently no high-level evidence available to support this notion. Therefore, we aimed to provide a comprehensive meta-analysis on all comparative studies regarding combination therapy of AC vs. AG in E. faecalis IE to further support therapeutic decisions in affected patients.

\section{Methods}

The study was conducted according to the current PRISMA guidelines [15], the principles of Good Clinical Practice and the Declaration of Helsinki. Prior to literature research, the study protocol was registered at the international prospective register of systematic reviews PROSPERO (ID: CRD42021268601) [16].

\section{Data sources and search strategy}

Literature search was performed by two independent reviewers (MM and LS) using predefined search terms ('endocarditis', 'aminoglycoside', 'gentamicin', 'beta lactam', 
'cephalosporin', 'ceftriaxone', 'penicillin', 'ampicillin', 'amoxicillin', 'enterococcus' and 'enterococ*') in the databases PubMed/MEDLINE, Cochrane Database of Systematic Reviews (CDSR), Cochrane Central Register of Controlled Trials (CENTRAL), Cochrane Clinical Answers (CCAs), EBM Reviews-Database of Abstracts of Reviews of Effects, Web of Science and Latin American and Caribbean Health Sciences Literature (LILACS) in June 2021. Additionally, manual search was performed by screening all references of eligible studies.

\section{Study selection}

Studies were considered eligible if $(\mathrm{P})$ patients included were $\geq 18$ years of age and had infective endocarditis with Enterococcus faecalis, (I) treatment with ampicillin-ceftriaxone (AC) was compared to $(\mathrm{C})$ ampicillin-gentamicin $(\mathrm{AG})$ and $(\mathrm{O})$ outcomes on in-hospital mortality, nephrotoxicity and adverse events requiring drug withdrawal were reported. Exclusion criteria were: studies including patients $<18$ years of age, studies on prophylactic treatment, animal studies or in vitro studies, studies on blood culture negative infective endocarditis (BCIEN), studies where data could not reliably be extracted, overlapping data, abstract-only papers, conference papers, editorials, books, articles without available full text, case reports and case series, as well as systematic reviews. No limits were set regarding the language and publication date of studies. Infective endocarditis was defined by the modified Duke criteria [17].

\section{Outcomes}

The primary outcome was in-hospital mortality. Secondary outcome measures were 3-month mortality, nephrotoxicity, adverse effects requiring drug withdrawal, relapse within 3 months of follow-up (defined as isolation of E. faecalis in blood specimens within the follow-up period) and treatment failure requiring change of antibiotic regimen.

\section{Statistical analyses}

Statistical analyses were performed with $\mathrm{R}$ (version 4.0.2., R Core Team (2013), R Foundation for Statistical Computing, Vienna, Austria; http://www.R-project.org/) using the packages 'Rcmdr', 'ggplot2', 'meta', 'metafor', 'dplyr' and 'dmetar'. Odds ratios and 95\% confidence intervals (CI) were calculated using random-effects models with the Mantel-Haenszel method, the Sidik-Jonkman estimator for $\tau^{2}$ and the Hartung-Knapp adjustment. Continuity correction was performed with treatment arm continuity correction (TACC). Between-study heterogeneity was assessed by calculating $\tau^{2}$, Higgin's \&Thompson's $I^{2}$, Cochran's $Q$, prediction intervals and by Baujat plot. Risk of bias assessment was conducted using the rob.summary() function of the 'dmetar' package for R, which is based on the Cochrance Risk of Bias Tool. A $p$ value $<0.05$ was considered statistically significant.

\section{Results}

\section{Selected studies}

Figure 1 depicts the PRISMA flow diagram [15] of literature search and study selection of our meta-analysis. After duplicate removal, 508 of the previously identified records were screened, which led to eligibility assessment of 7 studies $[12,13,18-22]$. Of these, 3 studies had to be excluded due to overlapping data $(n=1)$ [13], use of other antibiotic regimens than defined in the inclusion criteria $(n=2)[21$, $22]$ or inclusion of patients $<18$ years of age $(n=1$, see Fig. 1) [21]. Characteristics of the 4 included retrospective, nonrandomized, observational cohort studies [12, 18-20] are depicted in Table 1. A summary of the risk of bias assessment is depicted in Supplementary Fig. 1. Of note, we observed no substantial between-study heterogeneity (see Fig. 2a-c and Supplementary Fig. 2).

\section{Dosage and duration of antibiotic therapy}

Of the 4 included studies, only 3 [12, 18, 20] reported the exact dosage and duration of antibiotic treatment (see Table 1). These studies investigated patients who received either AC [ampicillin (2 g/4 h) + ceftriaxone (2 g/12 h)] or $\mathrm{AG}$ [ampicillin + gentamicin (3 mg/kg/day)] for 4-6 weeks; however, differences were reported in the frequency of gentamicin application (1-3 doses/day).

\section{In-hospital mortality and 3-month mortality}

Data on the primary outcome of in-hospital mortality were available from all 4 studies, whereas data on the secondary outcome of 3-month mortality were available from 3 studies only $[12,19,20]$. There was no statistically significant difference in in-hospital mortality or 3-month mortality between the two treatment arms (in-hospital mortality: OR 0.94 [0.56-1.59], $p=0.740$, see Figs. 2a and 3a; 3-month mortality: OR 1.31 [0.94-1.84], $p=0.072$ ).

\section{Nephrotoxicity}

Data on nephrotoxicity were available from all 4 studies (see Table 1). Of both treatment arms, nephrotoxicity occurred significantly less often in patients receiving $\mathrm{AC}$ than in patients treated with AG (OR 0.45 [0.26-0.77], $p=0.0182$, see Figs. $2 b$ and $3 b)$. 
Fig. 1 PRISMA flow diagram [15] of literature search and study selection

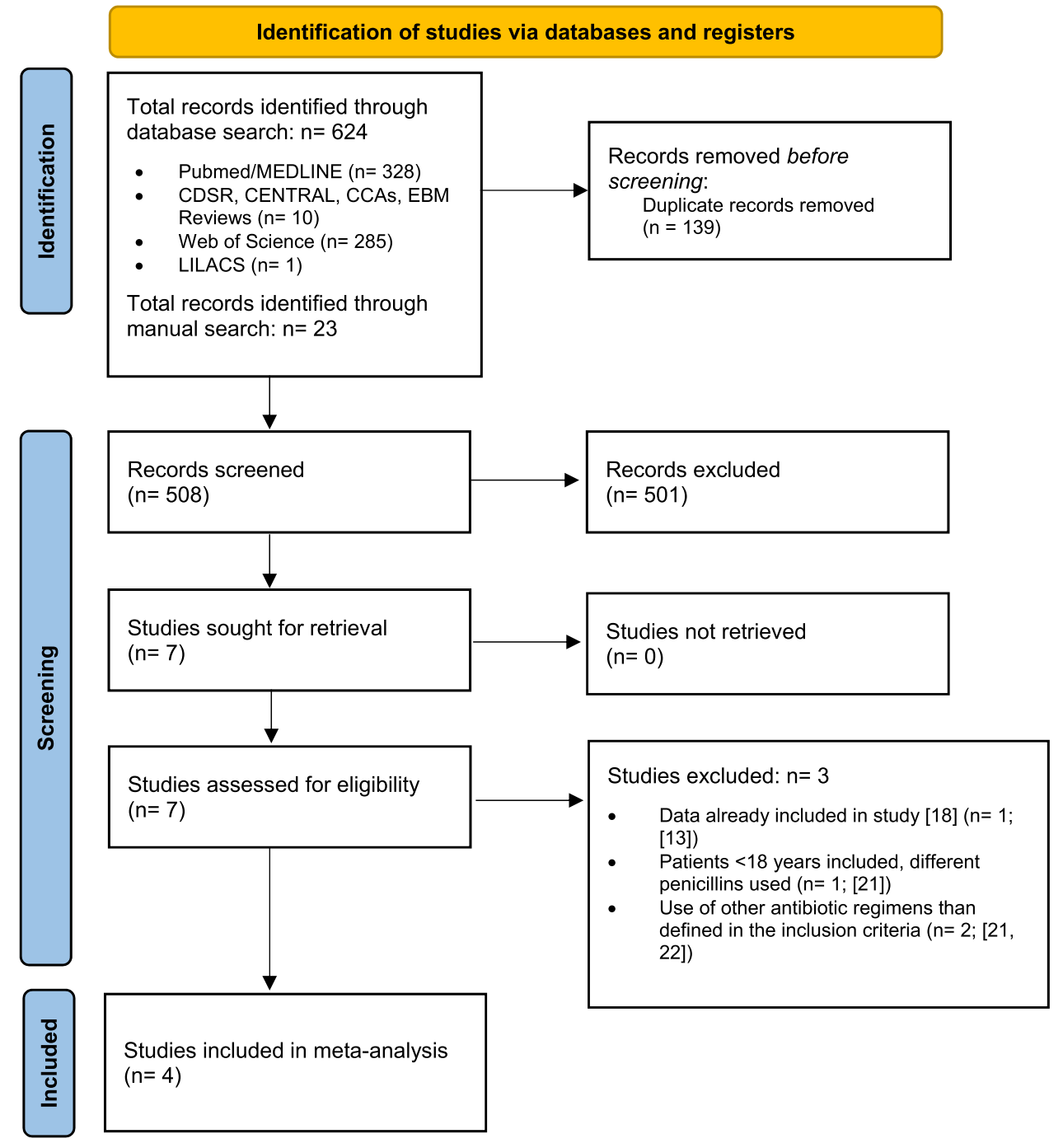

\section{Adverse events requiring drug withdrawal}

Data on drug withdrawal due to adverse events of antibiotic treatment were available from all 4 studies. Drug withdrawal occurred significantly less often in patients treated with AC than in patients treated with AG (OR 0.11 [0.03-0.46], $p=0.0160$, see Figs. $2 \mathrm{c}$ and $3 \mathrm{c}$ ).

\section{Relapse within 3 months of follow-up and treatment failure requiring change of antibiotic regimen}

Data on relapses were reported in 3 studies [12, 18, 20], whereas data on treatment failure were reported in 2 studies $[12,19]$. There were no statistically significant differences in these secondary outcome measures between the two treatment arms investigated (relapses: OR 1.21 [0.07-21.47], $p=0.8066)$; treatment failure: OR 0.47 [0.03-8.16], $p=0.1847)$.

\section{Discussion}

Infective endocarditis (IE) remains one of the most challenging diseases in modern cardiology. With an ever-increasing number of cardiac and extracardiac devices implanted, as well as an increasingly old and multimorbid population, the incidence of endocarditis has been slightly rising in recent years in parts of the world [23-28]. Furthermore, despite the availability of clinical practice guidelines and improvements in therapy itself, mortality of IE remains high with an estimated 12-month mortality rate of $15-30 \%$ reported in most studies [3, 27, 29]. Besides morbidity and mortality exerted by the infection itself, the intensive and tedious antibiotic treatment regimens of IE are in part associated with an increased risk for adverse outcomes, such as druginduced nephrotoxicity or ototoxicity in patients treated with aminoglycosides, rendering endocarditis and its necessary therapies a potentially debilitating disease entity for affected patients [30]. 


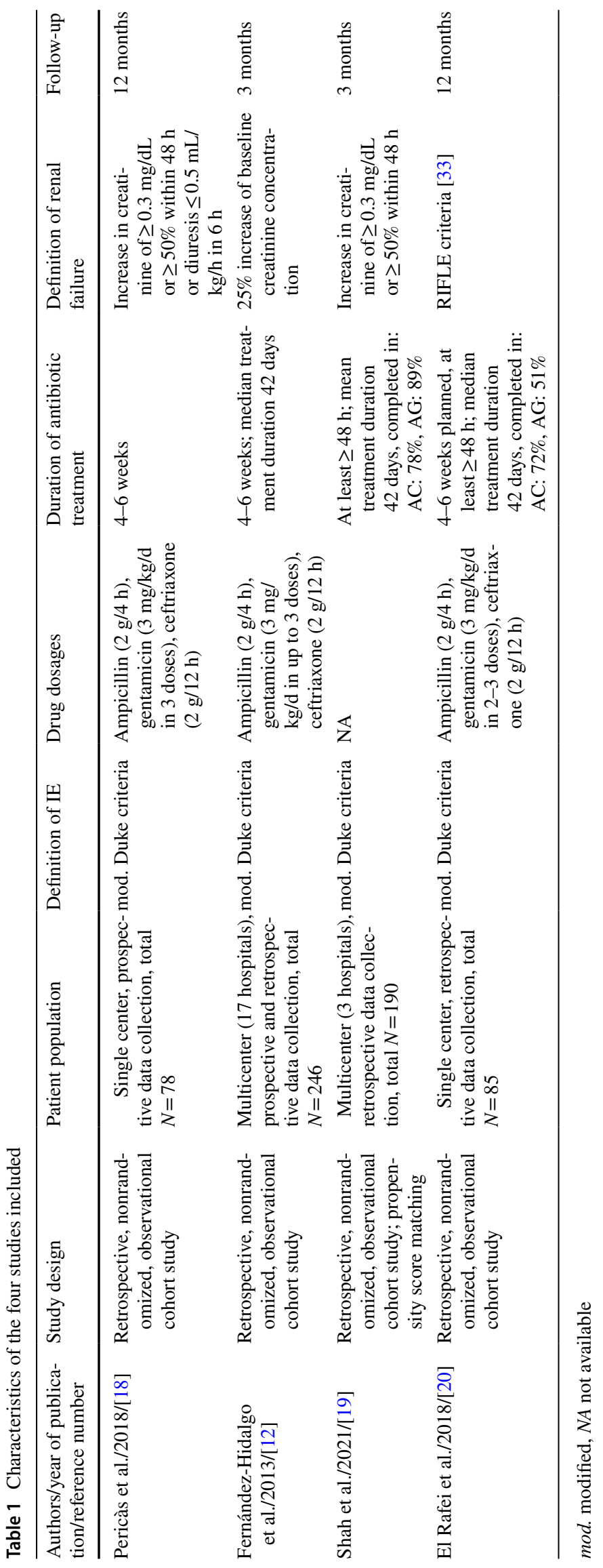




\section{a In-hospital mortality}

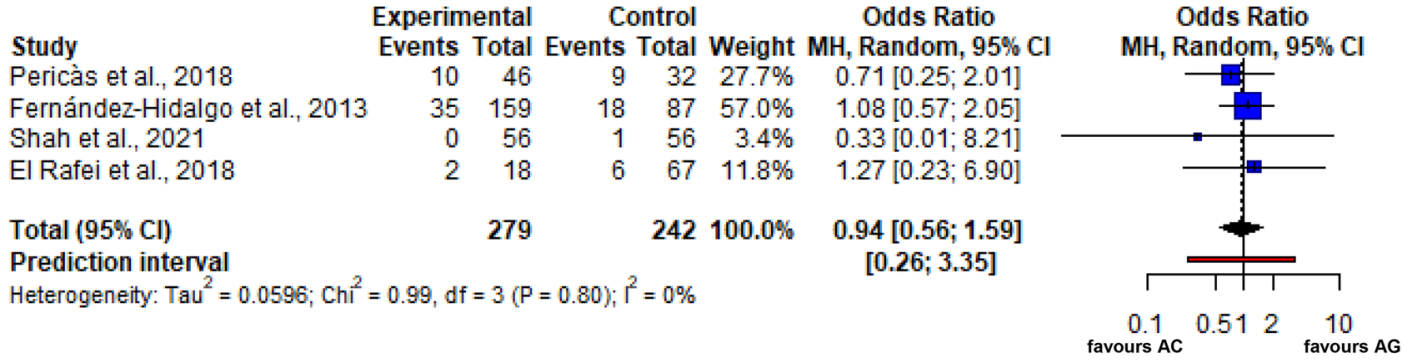

\section{b Nephrotoxicity}

\section{Study}

Pericàs et al., 2018

Fernández-Hidalgo et al., 2013

Shah et al., 2021

El Rafei et al., 2018

Total $(95 \% \mathrm{Cl})$

Prediction interval

Heterogeneity: $\operatorname{Tau}^{2}=0.0280 ; \mathrm{ChI}^{2}=1.93, \mathrm{df}=3(\mathrm{P}=0.59) ; \Gamma^{2}=0 \%$

323
Experimental Control Odds Ratio

Events Total Events Total Weight MH, Random, $95 \% \mathrm{Cl}$

$\begin{array}{llllll}15 & 46 & 20 & 32 & 20.8 \% & 0.29\end{array}[0.11 ; 0.75]$

$\begin{array}{llllll}53 & 159 & 40 & 87 & 52.8 \% & 0.59[0.34 ; 1.00]\end{array}$

$\begin{array}{llllll}6 & 100 & 13 & 90 & 18.3 \% & 0.38[0.14 ; 1.04]\end{array}$

$\begin{array}{llllll}2 & 18 & 17 & 67 & 8.1 \% & 0.37[0.08 ; 1.77]\end{array}$

$276100.0 \%$

$0.45[0.26 ; 0.77]$

$[0.16 ; 1.26]$

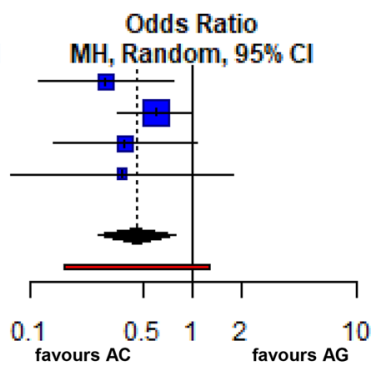

\section{Adverse events requiring drug withdrawal}

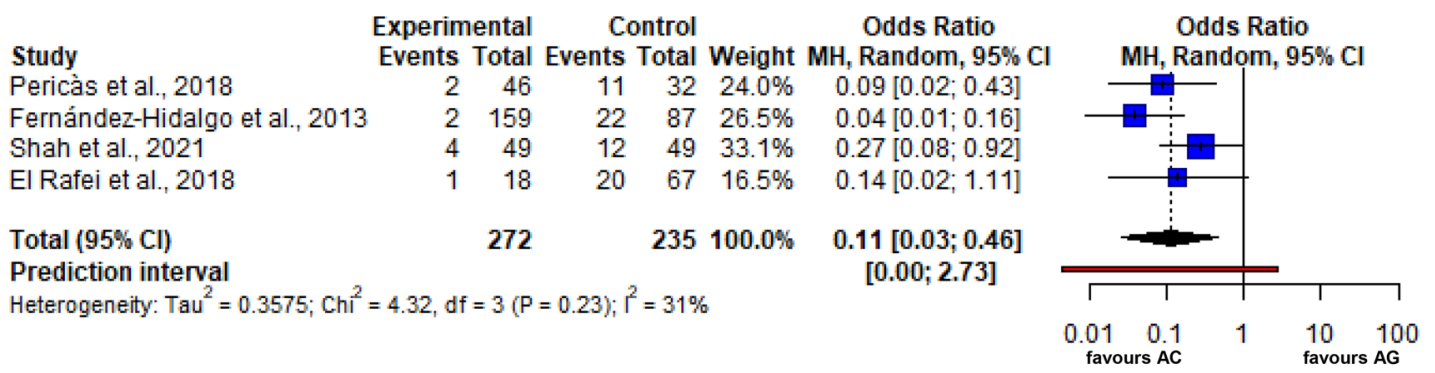

Fig. 2 Forest plots of a the primary outcome measure 'in-hospital mortality', b the secondary outcome measure 'nephrotoxicity' and c the secondary outcome measure 'adverse events requiring drug with-

Currently, guidelines by the European Society of Cardiology (ESC) recommend either a combination of ampicillin plus ceftriaxone (AC) or amoxicillin/ampicillin plus gentamicin (AG) in the treatment of Enterococcus faecalis infective IE [3]. However, among the aforementioned antibiotic drugs, gentamicin is associated with the highest risk of drug-induced adverse events, including potentially irreversible complications such as vestibulotoxicity or ototoxicity $[7,8]$. As guidelines currently state an equivalent class IB recommendation for both antibiotic regimens [3], the aim of this meta-analysis was to investigate the therapeutic non-inferiority of AC versus $\mathrm{AG}$, and to elucidate whether $\mathrm{AC}$ would be associated with a lower risk of drug-induced adverse events. drawal'. Depicted are the pooled effect estimate (dotted black line), the $95 \%$ confidence interval (CI; black diamond) and the prediction interval (red bar)

After including four retrospective, nonrandomized, observational cohort studies $[12,18-20]$ in this meta-analysis, we found that the $\mathrm{AC}$ regime was indeed non-inferior to $\mathrm{AG}$ in E. faecalis endocarditis, as depicted by no statistically significant differences in hospital mortality $(p=0.740), 3$-month mortality $(p=0.072)$, relapses $(p=0.8066)$ or treatment failure ( $p=0.1847)$ between the two treatment arms investigated. Furthermore, the presumed lower toxicity of the AC regime was confirmed by a significantly lower prevalence of nephrotoxicity (OR 0.45 [0.26-0.77], $p=0.0182$ ) and drug withdrawal due to adverse events (OR 0.11 [0.03-0.46], $p=0.0160$ ) when compared to patients receiving AG.

Our findings, showing non-inferiority of the AC regime, as well as a significantly reduced risk of drug-induced 

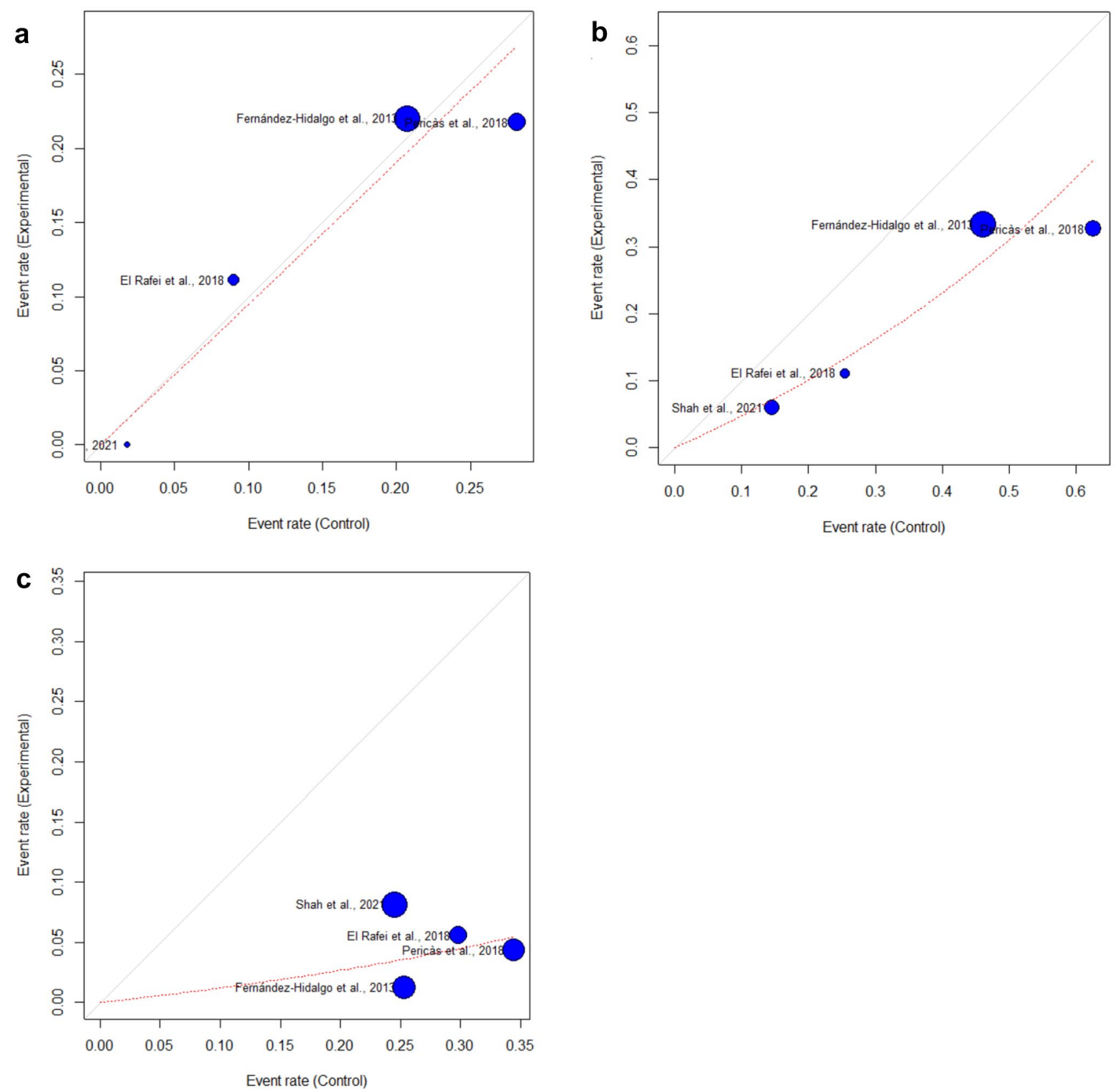

Fig. 3 L'Abbé plots of a the primary outcome measure 'in-hospital mortality', b the secondary outcome measure 'nephrotoxicity' and c the secondary outcome measure 'adverse events requiring drug with-

drawal'. Depicted are event rates of experimental treatment arm (AC) and control treatment arm (AG), as well as the pooled effect estimate (dotted red line)

adverse events, give rise to speculation that $\mathrm{AC}$ might be favourable over AG for affected patients with E. faecalis endocarditis. Especially in highlight of an increasingly old and multimorbid population, with a steadily incrementing prevalence of chronic kidney disease [31], the aforementioned findings should be taken into consideration when clinical decisions on antibiotic treatment in enterococcal IE are to be made. Moreover, the fact that high-level aminoglycoside-resistant (HLAR) strains of Enterococci increasingly

limit the use of AG in IE [6, 32] further strengthens the notion to abandon $\mathrm{AG}$ in favour of $\mathrm{AC}$ in this disease entity.

However, while exhibiting a good tolerability profile [33] and broad antimicrobial spectrum, treatment with ceftriaxone was previously identified as a major risk factor for Clostridium difficile infection, as well as for colonization with vancomycin-resistant E. faecium (VRE) or other multi-resistant bacteria [34-37]. The link to colonization with these pathogens, and its potential implications 
for the individual patient, as well as the patient's environment, should be considered when therapeutic decisions on antimicrobial treatment in IE are to be made.

Furthermore, both AC and AG are currently implemented as therapeutic regimens administered via repeated intravenous infusions. However, the Partial Oral Treatment of Endocarditis (POET) trial [38] recently found that a change to oral antibiotics after an initial intravenous phase was non-inferior to continued intravenous treatment in patients with IE. Since a reduction of hospital stay diminishes the risk of complications, associated costs, as well as psychological distress for our patients [38], outpatient treatment appears to be a promising opportunity in patients with E. faecalis IE as well. However, treatment with cephalosporins was not investigated in the POET trial, and bioavailability of these antibiotic drugs is unfortunately comparatively low [39]. Nonetheless, novel formulations are being investigated at the moment, which could help to put outpatient treatment with AC in patients with E. faecalis IE into practice in the near future $[40,41]$.

Finally, other antibiotic regimens are also depicted in the ESC guidelines on enterococcal IE, and can thus also be considered in affected patients [3]. However, vancomycin with gentamicin has a weaker class IC recommendation than $\mathrm{AC}$ or $\mathrm{AG}$, and treatment with daptomycin is currently recommended in patients with strains resistant to aminoglycosides, beta-lactams and vancomycin only. Furthermore, a recent study by Cerón et al. showed worse microbiological and clinical responses in patients treated with daptomycin when compared to standard treatment [22].

In conclusion, treatment with $\mathrm{AC}$ was non-inferior to treatment with $\mathrm{AG}$, and it was associated with a reduced prevalence of nephrotoxicity and drug withdrawal due to adverse events. Thus, combination therapy with AC appears favourable over AG in patients with E. faecalis IE.

\section{Conclusions}

Treatment with ampicillin plus ceftriaxone (AC) was noninferior to ampicillin plus gentamicin (AG) in E. faecalis endocarditis, as depicted by no statistically significant differences in-hospital mortality, 3-month mortality, relapses or treatment failure. Furthermore, AC was associated with a lower prevalence of nephrotoxicity and drug withdrawal due to adverse than AG. Given that it was non-inferior and associated with a reduced risk for drug-induced adverse events, AC might thus be favourable over AG in patients with E. faecalis IE.

\section{Limitations}

Our study has several limitations. First, all four included studies were relatively small, resulting in a total $N$ of only 599 patients included in the current meta-analysis. The low number of included patients, as well as the potential statistical implications thereof, has to be considered when interpreting the results of our study. Moreover, the definition of acute renal failure was slightly different between included studies (also see Table 1), which is why a certain bias on the secondary outcome measure 'nephrotoxicity' cannot be excluded. Of note, we only included data of patients with creatinine increase $\geq 50 \%$ from the study by El Rafei et al. [20, 42] to meet the definitions of the other included studies. In one study, the definition of acute renal failure was initially missing [19]; however, the corresponding author could provide the definition used when contacted.

Also, between-study heterogeneity was assessed by calculating $\tau^{2}, I^{2}, Q$ and prediction intervals. Here, we found no substantial heterogeneity; however, a low number of included studies $(k=4)$ can result in a bias of heterogeneity measures $[43,44]$, which is a known problem of small meta-analyses. Hence, a certain degree of betweenstudy heterogeneity cannot certainly be excluded (see also Supplementary Fig. 2). Also, a low $k$ is a problem when assessing publication bias, especially when small study effects should be calculated. Hence, a certain degree of publication bias can also not certainly be excluded. However, for visual estimation, contour-enhanced funnel plots are additionally depicted in Supplementary Fig. 3. The possible biases arising from a low number of included studies should be considered when interpreting the findings of our meta-analysis.

Supplementary Information The online version contains supplementary material available at https://doi.org/10.1007/s00392-021-01971-3.

Acknowledgements The Graphical Abstract was generated using Biorender.com.

Author contributions MM concepted and designed the study. MM and LS performed literature search and data collection, whereas MM conducted statistical analyses. MM, AT, and LS wrote the manuscript. $\mathrm{UH}$ and ML reviewed the paper and provided substantial improvements prior to submission. All authors read the final version of the manuscript and agreed to its contents.

Funding Open access funding provided by Paracelsus Medical University. No external funding was involved in the realization of this project.

Data availability The data underlying this study will be shared upon reasonable request to the corresponding author.

Code availability Not applicable. 


\section{Declarations}

Conflict of interest The authors declare that there are no conflicts of interest regarding the publication of this paper.

Ethics approval Not applicable.

Consent to participate Not applicable.

Consent for publication Not applicable.

Open Access This article is licensed under a Creative Commons Attribution 4.0 International License, which permits use, sharing, adaptation, distribution and reproduction in any medium or format, as long as you give appropriate credit to the original author(s) and the source, provide a link to the Creative Commons licence, and indicate if changes were made. The images or other third party material in this article are included in the article's Creative Commons licence, unless indicated otherwise in a credit line to the material. If material is not included in the article's Creative Commons licence and your intended use is not permitted by statutory regulation or exceeds the permitted use, you will need to obtain permission directly from the copyright holder. To view a copy of this licence, visit http://creativecommons.org/licenses/by/4.0/.

\section{References}

1. Murdoch DR, Corey GR, Hoen B et al (2009) Clinical presentation, etiology, and outcome of infective endocarditis in the $21 \mathrm{st}$ century: the international collaboration on endocarditis-prospective cohort study. Arch Intern Med 169:463-473. https://doi.org/ 10.1001/ARCHINTERNMED.2008.603

2. Chirouze C, Athan E, Alla F et al (2013) Enterococcal endocarditis in the beginning of the 21st century: analysis from the International Collaboration on Endocarditis-Prospective Cohort Study. Clin Microbiol Infect 19:1140-1147. https://doi.org/10. 1111/1469-0691.12166

3. Habib G, Lancellotti P, Antunes M et al (2015) 2015 ESC Guidelines for the management of infective endocarditis: The Task Force for the Management of Infective Endocarditis of the European Society of Cardiology (ESC). Endorsed by: European Association for Cardio-Thoracic Surgery (EACTS), the European. Eur Heart J 36:3075-3123. https://doi.org/10.1093/EURHEARTJ/ EHV319

4. Fernández-Hidalgo N, Almirante B, Tornos P et al (2012) Immediate and long-term outcome of left-sided infective endocarditis. A 12-year prospective study from a contemporary cohort in a referral hospital. Clin Microbiol Infect. https://doi.org/10.1111/ 1469-0691.12033

5. Dahl A, Rasmussen R, Bundgaard H et al (2013) Enterococcus faecalis infective endocarditis: a pilot study of the relationship between duration of gentamicin treatment and outcome. Circulation 127:1810-1817. https://doi.org/10.1161/CIRCULATIO NAHA.112.001170

6. Lebeaux D, Fernández-Hidalgo N, Pilmis B et al (2020) Aminoglycosides for infective endocarditis: time to say goodbye? Clin Microbiol Infect 26:723-728

7. Mingeot-Leclercq M-P, Tulkens PM (1999) Aminoglycosides: nephrotoxicity. Antimicrob Agents Chemother 43:1003

8. East JE, Foweraker JE, Murgatroyd FD (2005) Gentamicin induced ototoxicity during treatment of enterococcal endocarditis: resolution with substitution by netilmicin. Heart. https://doi.org/ 10.1136/hrt.2003.028308
9. Ahmed RM, Hannigan IP, MacDougall HG et al (2012) Gentamicin ototoxicity: a 23-year selected case series of 103 patients. Med J Aust 196:701-704. https://doi.org/10.5694/MJA11.10850

10. Storer AFB (2013) Gentamicin dosing and monitoring challenges in end-stage renal disease. Adv Pharmacoepidemiol Drug Saf. https://doi.org/10.4172/2167-1052.1000135

11. Mullins R, Lampasona V, Conn R (1987) Monitoring aminoglycoside therapy. Clin Lab Med 7:513-529

12. Fernández-Hidalgo N, Almirante B, Gavaldà J et al (2013) Ampicillin plus ceftriaxone is as effective as ampicillin plus gentamicin for treating Enterococcus faecalis infective endocarditis. Clin Infect Dis 56:1261-1268. https://doi.org/10.1093/cid/cit052

13. Pericas JM, Cervera C, del Rio A et al (2014) Changes in the treatment of Enterococcus faecalis infective endocarditis in Spain in the last 15 years: from ampicillin plus gentamicin to ampicillin plus ceftriaxone. Clin Microbiol Infect 20:O1075-O1083. https:// doi.org/10.1111/1469-0691.12756

14. Gavaldà J, Len O, Miró JM et al (2007) Brief communication: treatment of Enterococcus faecalis endocarditis with ampicillin plus ceftriaxone. Ann Intern Med 146:574-579. https://doi.org/ 10.7326/0003-4819-146-8-200704170-00008

15. Page MJ, McKenzie JE, Bossuyt PM et al (2021) The PRISMA 2020 statement: an updated guideline for reporting systematic reviews. BMJ. https://doi.org/10.1136/BMJ.N71

16. NIHR - National Institute for Health Research; Centre for Reviews and Dissemination; University of York PROSPERO - international prospective register of systematic reviews. https://www.crd.york. ac.uk/PROSPERO/. Accessed $18 \mathrm{Jul} 2021$

17. Li J, Sexton D, Mick N et al (2000) Proposed modifications to the Duke criteria for the diagnosis of infective endocarditis. Clin Infect Dis 30:633-638. https://doi.org/10.1086/313753

18. Pericàs JM, Cervera C, Moreno A et al (2018) Outcome of Enterococcus faecalis infective endocarditis according to the length of antibiotic therapy: preliminary data from a cohort of 78 patients. PLoS ONE. https://doi.org/10.1371/journal.pone.0192387

19. Shah NH, Shutt KA, Doi Y (2021) Ampicillin-ceftriaxone vs ampicillin-gentamicin for definitive therapy of Enterococcus faecalis infective endocarditis: a propensity score-matched, retrospective cohort analysis. Open Forum Infect Dis. https://doi.org/ 10.1093/ofid/ofab102

20. El Rafei A, DeSimone DC, Narichania AD et al (2018) Comparison of dual $\beta$-lactam therapy to penicillin-aminoglycoside combination in treatment of Enterococcus faecalis infective endocarditis. J Infect 77:398-404. https://doi.org/10.1016/j.jinf.2018.06. 013

21. Briggs S, Broom M, Duffy E et al (2021) Outpatient continuousinfusion benzylpenicillin combined with either gentamicin or ceftriaxone for enterococcal endocarditis. J Antimicrob Chemother. https://doi.org/10.1093/jac/dkab132

22. Cerón I, Muñoz P, Marín M et al (2014) Efficacy of daptomycin in the treatment of enterococcal endocarditis: a 5 year comparison with conventional therapy. J Antimicrob Chemother 69:16691674. https://doi.org/10.1093/jac/dku004

23. Holland T, Baddour LM, Bayer AS et al (2016) Infective endocarditis. Nat Rev Dis Prim. https://doi.org/10.1038/NRDP.2016.59

24. Forestier E, Fraisse T, Roubaud-Baudron C et al (2016) Managing infective endocarditis in the elderly: new issues for an old disease. Clin Interv Aging 11:1199-1206. https://doi.org/10.2147/ CIA.S 101902

25. Dayer M, Jones S, Prendergast B et al (2015) Incidence of infective endocarditis in England, 2000-13: a secular trend, interrupted time-series analysis. Lancet (London, England) 385:1219-1228. https://doi.org/10.1016/S0140-6736(14)62007-9

26. Thornhill M, Gibson T, Cutler E et al (2018) Antibiotic prophylaxis and incidence of endocarditis before and after the 2007 AHA 
recommendations. J Am Coll Cardiol 72:2443-2454. https://doi. org/10.1016/J.JACC.2018.08.2178

27. Cuervo G, Escrihuela-Vidal F, Gudiol C, Carratalà J (2021) Current challenges in the management of infective endocarditis. Front Med. https://doi.org/10.3389/FMED.2021.641243

28. Slipczuk L, Codolosa JN, Davila CD et al (2013) Infective endocarditis epidemiology over five decades: a systematic review. PLoS ONE 8:82665. https://doi.org/10.1371/JOURNAL.PONE. 0082665

29. Wallace S, Walton B, Kharbanda R et al (2002) Mortality from infective endocarditis: clinical predictors of outcome. Heart 88:53-60. https://doi.org/10.1136/HEART.88.1.53

30. Cahill T, Baddour LM, Habib G et al (2017) Challenges in infective endocarditis. J Am Coll Cardiol 69:325-344. https://doi.org/ 10.1016/J.JACC.2016.10.066

31. Lv J, Zhang L (2019) Prevalence and disease burden of chronic kidney disease. Adv Exp Med Biol 1165:3-15. https://doi.org/10. 1007/978-981-13-8871-2_1

32. Chow J (2000) Aminoglycoside resistance in enterococci. Clin Infect Dis 31:586-589. https://doi.org/10.1086/313949

33. Lamb H, Ormrod D, Scott L, Figgitt D (2002) Ceftriaxone: an update of its use in the management of community-acquired and nosocomial infections. Drugs 62:1041-1089. https://doi.org/10. 2165/00003495-200262070-00005

34. Beganovic M, Luther MK, Rice LB et al (2018) A review of combination antimicrobial therapy for Enterococcus faecalis bloodstream infections and infective endocarditis. Clin Infect Dis 67:303-309. https://doi.org/10.1093/cid/ciy064

35. McKinnell JA, Kunz DF, Chamot E et al (2012) Association between vancomycin-resistant enterococci bacteremia and ceftriaxone usage. Infect Control Hosp Epidemiol 33:718-724. https:// doi.org/10.1086/666331
36. Owens RC, Donskey CJ, Gaynes RP et al (2008) Antimicrobialassociated risk factors for Clostridium difficile infection. Clin Infect Dis 46:19

37. Dancer SJ (2001) The problem with cephalosporins. J Antimicrob Chemother 48:463-478. https://doi.org/10.1093/jac/48.4.463

38. Iversen K, Ihlemann N, Gill S et al (2019) Partial oral versus intravenous antibiotic treatment of endocarditis. N Engl J Med. https://doi.org/10.1056/NEJMOA1808312

39. Bergan T (1987) Pharmacokinetic properties of the cephalosporins. Drugs 34:89-104. https://doi.org/10.2165/00003495-19870 0342-00008

40. Maghrabia A, Boughdady M, Meshali M (2021) Design and optimization of new enteric nanoparticles of ceftriaxone for oral delivery: in vitro and in vivo assessments. Int J Nanomed 16:5937-5953. https://doi.org/10.2147/IJN.S319176

41. Lee S, Kim SK, Lee DY et al (2006) Pharmacokinetics of a new, orally available ceftriaxone formulation in physical complexation with a cationic analogue of bile acid in rats. Antimicrob Agents Chemother 50:1869-1871. https://doi.org/10.1128/AAC.50.5. 1869-1871.2006

42. Bellomo R, Ronco C, Kellum J et al (2004) Acute renal failure definition, outcome measures, animal models, fluid therapy and information technology needs: the Second International Consensus Conference of the Acute Dialysis Quality Initiative (ADQI) Group. Crit Care. https://doi.org/10.1186/CC2872

43. von Hippel P (2015) The heterogeneity statistic I(2) can be biased in small meta-analyses. BMC Med Res Methodol. https://doi.org/ 10.1186/S12874-015-0024-Z

44. IntHout J, Ioannidis J, Rovers M, Goeman J (2016) Plea for routinely presenting prediction intervals in meta-analysis. BMJ Open. https://doi.org/10.1136/BMJOPEN-2015-010247 